\title{
Feasibility and effect of life skills building education and multiple micronutrient supplements versus the standard of care on anemia among non-pregnant adolescent and young Pakistani women (15-24 years): a prospective, population- based cluster-randomized trial
}

Jo-Anna B. Baxter ${ }^{1,2}$, Yaqub Wasan³ , Sajid B. Soofi ${ }^{3}$, Zamir Suhag ${ }^{3}$ and Zulfiqar A. Bhutta ${ }^{1,2,3^{*}}$

\begin{abstract}
Background: Adolescence is a critical period for physical and psychological growth and development, and vitamin and mineral requirements are correspondingly increased. Health and health behaviours correspond strongly from adolescence to adulthood. Developing a preconception care package for adolescent and young women in resource-limited settings could serve to empower them to make informed decisions about their nutrition, health, and well-being, as well as function as a platform for the delivery of basic nutrition-related interventions to address undernutrition.

Methods: In this population-based two-arm, cluster-randomized, controlled trial of life skills building education (provided bi-monthly) and multiple micronutrient supplementation (provided twice-weekly; UNIMMAP composition) , we aim to evaluate the effectiveness of the intervention on the prevention of anemia (hemoglobin concentration $<12 \mathrm{~g} / \mathrm{dL}$ ) among adolescent and young women (15-24 years) in Matiari district, Pakistan compared to the standard of care. Several secondary objectives related to nutrition (anthropometry [height, weight, middle upper arm circumference (MUAC)], nutritional status [iron, vitamin A, vitamin D]); general health (morbidity, mortality); and empowerment (age at marriage, completion of the 10th grade, use of personal hygienic materials during menstruation) will also be assessed. Participants will be enrolled in the study for a maximum of 2 years.

\footnotetext{
* Correspondence: zulfiqar.bhutta@aku.edu

${ }^{1}$ Centre for Global Child Health, The Hospital for Sick Children, Toronto, ON

M6S 156, Canada

${ }^{2}$ Department of Nutritional Sciences, University of Toronto, Toronto, ON,

Canada

Full list of author information is available at the end of the article
}

(C) The Author(s). 2018 Open Access This article is distributed under the terms of the Creative Commons Attribution 4.0 International License (http://creativecommons.org/licenses/by/4.0/), which permits unrestricted use, distribution, and reproduction in any medium, provided you give appropriate credit to the original author(s) and the source, provide a link to the Creative Commons license, and indicate if changes were made. The Creative Commons Public Domain Dedication waiver (http://creativecommons.org/publicdomain/zero/1.0/) applies to the data made available in this article, unless otherwise stated. 


\begin{abstract}
(Continued from previous page)
Discussion: Empowering adolescent and young women with the appropriate knowledge to make informed and healthy decisions will be key to sustained behavioural change throughout the life-course. Although multiple micronutrient deficiencies are known to exist among adolescent and young women in low-resource settings, recommendations on preconception multiple micronutrient supplementation do not exist at this time. This study is expected to offer insight into providing an intervention that includes both education and supplements to nonpregnant adolescent and young women for a prolonged duration of time within the existing public health programmatic context.

Trial registration: This study is part of the Matiari emPowerment and Preconception Supplementation (MaPPS) Trial. The MaPPS Trial was registered retrospectively on clinicaltrials.gov (Identifier: NCT03287882) on September 19, 2017.
\end{abstract}

Keywords: Adolescence, Young adult, Nutrition, Micronutrients, Education, Preconception, Anemia, Empowerment

\section{Plain English summary}

In low- and middle-income countries many adolescent and young women do not consume enough healthy and nutrient-rich foods to meet their daily needs. Data show that adolescent and young women in these places are also more likely to become pregnant. If a woman is undernourished when she becomes pregnant, it can have a negative effect on her health, pregnancy, and infant. One way to improve adolescent and young women's health before they become pregnant is to provide a program that aims to help them make healthy decisions and improve their nutrition. This is often called preconception care. In this study in rural Pakistan, we have designed a culturally-appropriate preconception care package that includes life skills building education, to help study participants make better decisions about their health and educate them about their rights, and multiple micronutrient supplements, since they have limited access to foods with the micronutrients that they need. Study participants (1524 years) are enrolled for 2 years, and we will assess several factors about their nutrition, health, and well-being over this time. The main effect we aim to determine is how many participants have anemia among those who receive the preconception care package compared to those who do not receive it. Ultimately, we hope that what we learn in this study will lead us to make recommendations about policies around preconception care for adolescent and young women so that they can live healthier lives and make healthier decisions.

\section{Background}

Within the Sustainable Development Goals, promoting strategies to ensure the survival, health, and well-being of adolescent girls, women, and their offspring has been recognized as an essential component to ending poverty [1]. Adolescence, in particular, is considered a neglected yet critical period for physical and psychological growth and development, given the transition from childhood to reproductive adulthood [2]. Pregnancies to adolescents are associated with a higher risk of complications and maternal and child mortality [3], and contribute to $23 \%$ of the burden of disease arising from pregnancy and childbirth [4]. When an adolescent becomes pregnant in a resourcelimited setting, her access to education generally stops as a result; yet, educating girls is very effective in raising overall economic productivity, lowering infant and maternal mortality, improving nutrition, and promoting health [5]. Collectively, this makes determining interventions to improve health outcomes from adolescence of public health importance [2].

Nutritional deficiencies are risk factors known to exist from adolescence, and have important consequences for future maternal and newborn health. During adolescence, vitamin and mineral requirements are incrementally increased to support one's ongoing physical development and the nutritional strain of menstruation [6]. Given the limited intake of animal products, fruits, vegetables, and fortified foods in low- and middle-income countries, undernutrition can be widely prevalent [7]. Deficiencies in multiple micronutrients (MMN) are of particular concern given their direct effects, such as iron deficiency anemia and iodine deficiency disorders, and their indirect effects, like increased risk of serious infectious disease [6]. The burden of undernutrition and its downstream consequences could be prevented throughout the life course by using appropriate nutrition-specific and nutrition-sensitive interventions, such as MMN supplementation and the empowerment of women to make informed decisions, respectively [5].

Since health and health behaviours correspond strongly from adolescence to adulthood, habits acquired during adolescence could have significant implications later in life [2]. As such, developing a preconception care package for adolescent and young women could serve to empower them to make informed decisions about their health and well-being [8], and function as a platform for the delivery of basic nutrition-related interventions to address undernutrition [9]. Incorporating a life skills building education (LSBE) element will be important to sustainable and proficient uptake in improving their own general health, 
pregnancy outcomes, and future health of their children [8]. However, at this time there is limited understanding as to what health information should be provided and how within preconception care.

We hypothesize that the programmatic provision of LSBE and MMN supplements to non-pregnant adolescent and young women 15-24 years of age in a population-based setting in rural Pakistan will improve selected nutrition, health, and empowerment-related outcomes compared to those who receive the standard of care. For those who receive the intervention, we expect that there will be a decrease in the prevalence of anemia. Conducting this trial within the context of the existing public health setting will allow us to assess the effectiveness of the intervention, and offer important insight around how to achieve coverage in the trial setting.

\section{Methods}

The Matiari emPowerment and Preconception Supplementation (MaPPS) Trial is a two-arm, cluster-randomized, controlled trial of LSBE and MMN supplementation provided in a programmatic context to evaluate the impact on pre-identified nutrition and health outcomes among adolescent and young women, and their infants born within the context of the trial. Depending on whether participants become pregnant or not, there are three phases: preconception (maximum 24 months in duration); pregnancy (approximately 9 months in duration); and postpartum (maximum 12 months in duration). A detailed trial protocol has been developed in accordance with the SPIRIT guidelines [10] in a collaboration between the Aga Khan University (AKU; Karachi, Pakistan) and the Hospital for Sick Children (Toronto, Ontario, Canada). This paper addresses the methods for ongoing data collection during the preconception phase of the trial among participants who do not become pregnant, with the methods for the parent trial detailing the pregnancy and postpartum phases appearing elsewhere ([11] unpublished).

The first participant was enrolled on 30 June 2017; enrollment is expected to be ongoing until July 2018; and planned data collection will continue until 2021.

\section{Objectives}

The primary aim of the preconception phase of the MaPPS Trial is to evaluate the impact of LSBE (provided bi-monthly) and supplementation with MMN (provided twice-weekly) versus the standard of care (non-regulated community-based health sessions and no supplements) on anemia (hemoglobin concentration $<12 \mathrm{~g} / \mathrm{dL}$ ) after 2 years among non-pregnant adolescents 15-18.9 years of age at enrolment. There are several secondary objectives to further determine the effect of LSBE and MMN supplementation compared to the standard of care.
These relate to nutrition (anthropometry [height, weight, middle upper arm circumference (MUAC)], nutritional status [iron, vitamin A, vitamin D]); general health (e.g., morbidity, mortality); and empowerment (age at marriage, completion of the 10th grade, and use of personal hygienic materials during menstruation).

\section{Setting and participants}

This trial will be conducted in rural settings within Matiari district in Sindh province, Pakistan. Briefly, the nutritional status of adolescent girls in Pakistan is poor, with 51 and 20\% experiencing anemia and iron deficiency anemia, respectively [12]. An urban/rural divide is suggested to impact adolescent nutritional status [13], with adolescents living in rural settings having particularly limited dietary diversity [14]. The intervention is situated within Pakistan's existing Lady Health Worker (LHW) Programme. Each LHW is affiliated with a health facility and visit the homes in her catchment area monthly to carry out community support sessions and disseminate health education messages [15]. LHW-led community sessions have the potential to serve as an important communication channel for adolescents, especially those out of school. However, the content of existing LHW educational materials primarily focuses on priority groups, including children $<5$ years, pregnant women, and couples eligible for family planning [16]. Although the LHW curriculum includes some material on topics important to the health of adolescent girls (e.g., dietary practices, anemia), these materials are not widely implemented and not included in community sessions regularly. More detail about the trial setting is described in the parent trial protocol publication ([11] unpublished).

\section{Eligibility criteria}

As the MaPPS Trial is a population-based effectiveness study of LSBE and MMN supplementation from preconception, broad eligibility criteria for participation in the preconception phase were established. These include that the minimum age at enrolment is 15 years and the maximum age is 23 years; adolescent and young women must also report to be physically able to comply with the trial intervention. Adolescent and young women are not eligible to enrol if they are pregnant, participating in a different nutrition trial, or intend to leave the study area. They can be of any marital status. If a participant becomes pregnant, she proceeds to the pregnancy phase of the MaPPS Trial, as detailed in the parent study publication ([11] unpublished).

The micronutrient status and dietary intake of a sub-sample of adolescent participants 15-18.9 years of age at enrolment will also be monitored within the MaPPS Trial. Participants recruited to the dietary 
assessment subgroup must also be a part of the micronutrient status subgroup.

\section{Design and sample size}

The MaPPS Trial is a two-arm, parallel, prospective, cluster-randomized, controlled trial (Fig. 1). A cluster-randomized design was chosen to prevent contamination between the control and intervention arms through supplement sharing. The unit of randomization is previously defined and mapped health facility clusters. A total of 26 clusters are available to be randomized (i.e., 13 clusters per arm), and more detail around randomization and allocation concealment are found with the description of the parent study (Baxter et al., unpublished). PASS 11 Software (NCSS, LLC., Kaysville, UT, USA) was used to determine all sample size calculations.

To observe a $20 \%$ relative reduction in the prevalence of anemia among non-pregnant adolescent participants 15-18.9 years at enrolment, a 50\% baseline prevalence of anemia [12] was assumed. With icc $=0.0256, \mathrm{k}=0.16$, accounting for $15 \%$ attrition, and probability of type I and type II errors of 0.05 and 0.20 , respectively, the number anemic adolescents required per cluster was 66 . This equates to 858 anemic adolescents per arm. To achieve this reduction in anemia, 1716 adolescents per arm will be required (3432 total). Given the number of adolescent and young women required to be powered to see a reduction in the primary outcome of the parent trial (low birth weight), we aim to enrol 12,712 participants $15-24$ years per arm $(25,424$ total).

To monitor the micronutrient status of a subgroup of participants 15-18.9 years of age at enrolment, the sample size calculated for the reduction in anemia prevalence will be used (i.e., 1716 adolescents per arm). Stata software (StataCorp, College Station, TX, USA) has been used to randomly select 132 adolescents $15-18.9$ years at enrolment from each cluster. Dietary intake will also be assessed in 200 randomly selected micronutrient status subgroup participants per arm (15 women per cluster), to further form a dietary status subgroup.

\section{Intervention and control LSBE materials}

In intervention clusters, enhanced LSBE materials have been developed on topics important to the empowerment of adolescent and young women to complement the existing LHW materials for use in community sessions. These will be implemented bi-monthly and attendance will be recorded. Three topic areas were prioritized: (a) delaying early marriage; (b) practicing appropriate personal and menstrual hygiene; and (c) the importance of good nutrition to health. Approximately $20 \mathrm{~min}$ is intended to be spent on each topic at community sessions. Integrated throughout the 3 topics are messages related to continuing one's education, mental health (how to cope with stress, anxiety, and when you are upset), gender norms and equality, decision-making, advocacy, resiliency, participation, communication skills, facing challenges, agency, conflict resolution, and the prevention of violence.

Communication tools have been developed to assist the LHWs in conducting the LSBE-based community sessions. These were designed in coordination with Aahung, a Karachi-based non-governmental organization that aims to improve the sexual and reproductive health of girls (www.aahung.org; Karachi, Pakistan). Communication tools include a flipchart with 2 pictorials and discussion prompts per topic for use in sessions; and a brief summary pamphlet for distribution to participants. The flipcharts are similar to existing LHW tools for leading community sessions. A team of master trainers is trained on all LSBE materials and appropriate communication techniques. The master trainers provide comprehensive and interactive training for all intervention LHWs. Refresher

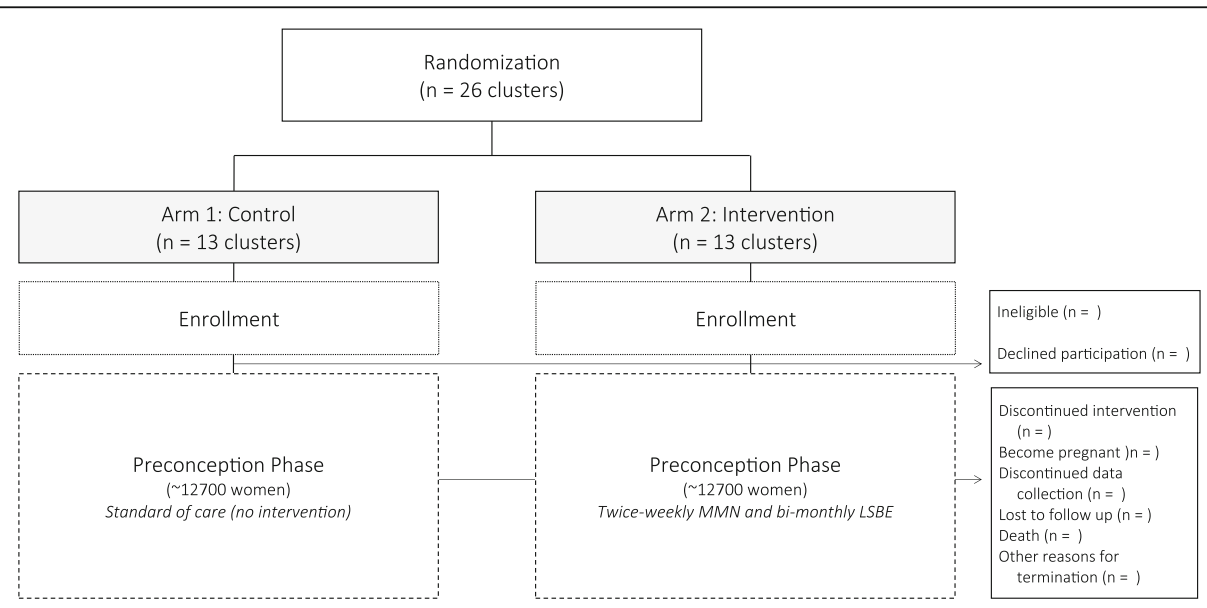

Fig. 1 Matiari emPowerment and Preconception Supplementation (MaPPS) Trial flow diagram for participants who do not become pregnant 
training will also be provided on an ongoing basis throughout the duration of the trial.

\section{Trial supplements and administration}

The MMN supplements provided to participants in the intervention arm of the MaPPS Trial are consistent with the UNICEF/WHO/UNU international multiple micronutrient preparation (UNIMMAP), and have been procured from the UNICEF Supply Catalogue (https://supply.unicef.org). Each tablet is $10 \mathrm{~mm}$ in diameter and includes vitamin A: $800 \mu \mathrm{g}$ RE; vitamin D: $5 \mu \mathrm{g}$; vitamin E: $10 \mathrm{mg}$; folic acid: $400 \mu \mathrm{g}$; vitamin B1 (thiamine): $1.4 \mathrm{mg}$; vitamin B2 (riboflavin): $1.4 \mathrm{mg}$; vitamin B3 (niacin): $18 \mathrm{mg}$; vitamin B12: $2.6 \mu \mathrm{g}$; vitamin B6: $1.9 \mathrm{mg}$; vitamin C: $70 \mathrm{mg}$; iron: $30 \mathrm{mg}$; zinc: $15 \mathrm{mg}$; iodine: $150 \mu \mathrm{g}$; copper: $2000 \mu \mathrm{g}$; selenium $65 \mu \mathrm{g}$ (Micronutrient Tabs, Pregnancy; Lomapharm, Emmerthal, Germany). All MMN supplements are maintained and stored by the study manager in a locked study office that is temperature controlled. MMN supplements are provided to LHWs monthly by a study monitoring team, which is unblinded to the allocation of the intervention and not involved in data collection. Participants' personal supply of MMN supplements are monitored and replenished every month by their respective LHWs. Once provided to participants, MMN supplements are stored in marked, opaque containers labelled with the participant's ID and supplement batch number and expiry date.

Upon enrolment in the trial, intervention arm LHWs will visit new participants within 1 month to provide MMN supplements. Participants are asked to consume 1 MMN tablet 2 days/week. To make the incorporation MMN supplementation into their routine easier, participants are requested to choose a consistent time on 2 days in the week that are separate from each other (e.g., Monday and Thursday, or Wednesday and Saturday) on which to take $1 \mathrm{MMN}$ tablet with a glass of water and a meal. Each participant is provided with a 1 month supply of MMN supplements, plus a spare week in case of unscheduled missed visits. As such, participants receive $10 \mathrm{MMN}$ tablets per month (4 weeks/month $\times 2$ tablets/week +2 extra tablets $=10$ tablets total). If a participant does not become pregnant within the context of the trial, she will take the MMN supplements for up to 24 months (the maximum duration of the preconception period).

Participants are instructed that the MMN supplements are just for them and should be kept away from children. They are asked to ensure that the lid of the supplement bottle is tightly closed when not in use and stored away from light and humidity. It is also explained that some people experience mild side effects when they start taking the MMN supplements (e.g., nausea, vomiting, diarrhea, stomach pains), but that taking it with food should make side effects less likely to happen. If they miss a dose, participants are instructed to take $1 \mathrm{MMN}$ tablet the next time that they remember, but never to consume more than 1 tablet/day. If a participant has any side effects, is concerned, or has questions, she is instructed to contact her LHW and not study personnel since study personnel are blinded to whether participants are in the control or intervention arm. Participants are also asked not to take non-trial administered supplements while they are enrolled in the trial.

\section{Enrolment and consent}

Adolescent and young women in LHW-covered areas in Matiari district were identified in a household listing exercise. Provisionally eligible participants will be invited to participate in MaPPS Trial with their family members and/or husbands present. Data collectors will explain the purpose and voluntary nature of the trial; participation components; and potential benefits and harms prior to obtaining written informed consent. If participants are < 16 years of age, assent will also be obtained as necessary. It is anticipated that enrolment will take at least 1 year.

\section{Visit schedule}

All visits with study participants will occur at their homes (Table 1). Upon enrolment in the trial, a questionnaire designed to collect information on demographics; socioeconomic status (SES); reproductive health and history; life skills, empowerment, and social determinants of health (SDH)-related factors (Table 2); supplementation practices; and access to LHWs will be administered. This questionnaire has been adapted from the existing demographic health survey for Pakistan and several standardized assessment tools. All participants will also undergo anthropometric (height, weight, MUAC) and hemoglobin concentration measurement. After 12 months of enrolment in the preconception phase, all participants' anthropometric measures (height, weight, MUAC) will be reassessed. After 24 months, all participants will be asked to complete a modified version of the baseline questionnaire for ongoing assessment of life skills, empowerment, and SDH-related factors; and undergo repeat anthropometric (height, weight, MUAC) and hemoglobin concentration measurements. Upon trial completion or withdrawal, participants will be asked to complete an exit questionnaire designed to assess their perception of their own health and satisfaction with trial participation.

Additional measures will be collected from participants in the micronutrient status subgroup, including a $5 \mathrm{~mL}$ blood draw at enrolment and after 24 months; repeat anthropometric assessment (height, weight, MUAC) after 6 months; and a modified version of the baseline questionnaire for ongoing assessment of life skills and empowerment at 12 months. Participants in the dietary status subgroup will complete 3 dietary assessments 
Table 1 Preconception phase data collection and measurement activities

\begin{tabular}{|c|c|c|c|c|}
\hline \multirow[t]{2}{*}{ Activity } & \multicolumn{4}{|c|}{ Month of participation } \\
\hline & 0 & 6 & 12 & 24 \\
\hline Consent & $x$ & & & \\
\hline Demographics and SES & $x$ & & M & $x$ \\
\hline Reproductive health and history & $x$ & & & \\
\hline Life skills, empowerment, and SDH & $x$ & & M & $x$ \\
\hline Anthropometry & $x$ & M & $x$ & $x$ \\
\hline Hemoglobin & $x$ & & M & $x$ \\
\hline Serum ferritin & M & & M & M \\
\hline Hepcidin & M & & M & M \\
\hline Transferrin receptor & M & & M & M \\
\hline Infection indicators (CRP, AGP) & M & & M & M \\
\hline Vitamin A & M & & M & M \\
\hline Vitamin D & M & & M & M \\
\hline Household food insecurity & $x$ & & M & $x$ \\
\hline Food group consumption survey (1 week) & $\mathrm{D}$ & & & \\
\hline 24-h recall & D & & & \\
\hline
\end{tabular}

$\mathrm{X}$ : all participants; $M$ micronutrient status subgroup participants only; $D$ dietary status subgroup participants only

(24-h food recall, weekly food group consumption survey) over the 3 weeks following enrolment in the trial. Dietary assessment days will be non-consecutive and unannounced, and include 2 weekdays and 1 weekend day.

\section{LHW visits}

Because LHWs are required to visit households in their catchment areas monthly, these visits will serve to monitor menses, new marriages, and suspected pregnancies throughout the duration of the trial, in addition to their mandated function [15]. All trial-administered supplement provision and consumption will also be recorded at these visits.

\section{Adherence, morbidity, and morbidity monitoring}

There will also be ongoing participant monitoring for the duration of the trial (Table 3). All participants will be visited by an independent surveillance team to collect morbidity and self-reported adherence data every 3 months. These visits will also allow for the observance and collection of data on non-trial administered nutritional supplement consumption.

\section{Data collection}

Data collection tools have been customized for each type of visit, and data is collected orally given the low literacy rates in the trial population. Trained study personnel will collect all trial outcome data using questionnaires, anthropometric measurements, point-of-care tests, and dietary recall methods. Standardized operating procedures have been developed for all measures in an effort to make data collection practices consistent. Extensive training sessions will be provided to study personnel, employing classroom-based lectures, videos, hands-on practice, and mock interviews.

\section{Questionnaires}

For data collector-conducted visits with participants, tablets (Samsung Galaxy Tab. A T285; Samsung, Vietnam) will be used to collect questionnaire data. The tablets run a custom-made data collection application, which includes built-in logic and range checks. LHW monitoring visits will use paper-based questionnaires given the large number of LHWs involved in the trial. Dietary assessments will also be paper-based.

\section{Anthropometric measurements}

To assess adolescent and young women's anthropometrics, height will be measured using a stadiometer (seca 213; seca, Hamburg, Germany); weight using a digital floor scale (seca 813); and MUAC using a measuring tape (seca 201). All measurements will be conducted in

Table 2 Factors relating to life skills, empowerment, and SDH captured within trial questionnaires

\begin{tabular}{lll}
\hline Domains & Includes information pertaining to & Evaluation tool \\
\hline $\begin{array}{l}\text { Nutrition } \\
\text { Health }\end{array}$ & Household food insecurity & Household Food Insecurity Access Scale [17] \\
& Eating habits & Health Behaviour in School-aged Children [18] \\
& Physical activity & \\
Perception of support & Family, peer, and school & Multidimensional Scale of Perceived Social Support [19] \\
Decision making & Family, food, healthcare, and daily needs & Pakistan Demographic Health Survey [20] \\
Psychosocial health & Self-efficacy & Generalized self-efficacy scale [21] \\
& Stress & Perceived stress scale [22] \\
Miolence & Mental health (depression, anxiety, and stress) & Depression, Anxiety, and Stress Scale-21 [23] \\
& Intimate partner violence & Conflict Tactics Scale [24] \\
\hline
\end{tabular}


Table 3 Monitoring data collection activities ongoing throughout the trial

\begin{tabular}{|c|c|c|}
\hline Measurement & Monthly (LHW) & Quarterly (Monitoring Team) \\
\hline Adherence by pill count ${ }^{\mathrm{a}}$ & i & \\
\hline Adherence by self-reported frequency ${ }^{b}$ & & i \\
\hline Side effects & & $x$ \\
\hline Supplement acceptability & & i \\
\hline Morbidity & & $x$ \\
\hline Mortality & & $x$ \\
\hline
\end{tabular}

$\mathrm{X}$ : all participants; i: intervention group only

${ }^{a}$ Adherence by pill count defined as number of tablets apparently consumed/number of possible supplements consumed given the number of days enrolled in the study

${ }^{\mathrm{b}}$ Adherence options by self-report during preconception include twice weekly, intermittently, or not at all

duplicate by 2 data collectors. In the case that 2 measurements exceed the pre-set allowable differences (height: $<1.0 \mathrm{~cm}$; weight: $<0.5 \mathrm{~kg}$; MUAC: $<0.5 \mathrm{~cm}$ ), a third measurement will be obtained. The average (mean) of acceptable paired measures will be used in analysis.

\section{Point-of-care hemoglobin assessment}

To assess hemoglobin concentration, the HemoCue ${ }^{\circ} \mathrm{Hb}$ 301 System (HemoCue; Ängelholm, Sweden) will be used from blood collected via finger prick.

\section{4- $h$ dietary recall}

Among participants enrolled in the dietary assessment subgroup, usual dietary intake will be assessed using an interactive 24-h recall method [25] administered by trained food recall data collectors. Consumed food portions will be estimated and identified using a locally developed food kit and food item reference manual [26].

\section{Blood specimen collection and laboratory analyses}

Trained phlebotomists (blinded to cluster allocation) will conduct all blood specimen collection using standard sampling procedures developed by the AKU Nutrition Research Lab. At each sampling time point, $5 \mathrm{~mL}$ of venous blood will be collected and stored in trace element-free vacutainer tubes (royal blue top; Greiner Bio-One, Monroe, NC, USA). Two drops of whole blood are immediately taken from the collection tube to assess hemoglobin concentration using the $\mathrm{HemoCue}^{\odot} \mathrm{Hb} 301$ System point of care test. Samples will be further assessed for markers of nutritional status (iron [ferritin, transferrin receptor, hepcidin], vitamin A [retinol], and vitamin D [25(OH)D]) and inflammation (c-reactive protein, alpha-1-acid glycoprotein). More details on blood specimen collection and assays can be found within the description of the parent trial (Baxter et al., unpublished).

\section{Data management}

Data collection tablets are collected from study personnel on a daily basis so that questionnaire data can be uploaded to the AKU data management unit.
Paper-based questionnaire forms are visually checked by field site supervisors for completeness before being sent to the AKU data management unit on a weekly basis for entry into a database. Double data entry is used to reduce data entry errors. All collected data is kept under lock and key, and anonymized through the use of nine-digit participant identification codes. Data entered into the database is password protected.

\section{Outcome measures}

To assess the prevalence of anemia, the primary outcome measures is hemoglobin concentration $<12 \mathrm{~g} / \mathrm{dL}$. There are many additional secondary outcomes within the trial related to health, nutrition, and empowerment that will be also assessed (see Additional file 1).

\section{Statistical analysis}

For the primary outcome, the prevalence of anemia will be compared by intervention arm, irrespective of preconception supplementation duration or adherence (intention-to-treat). The per-protocol analysis will consider those women who complied with the recommend MMN supplementation frequency. Because the prevalence of anemia is high in the trial population, an individual-level analysis using generalized estimating equations (GEE) will be employed to determine the estimate of the population average. Clustering will be accounted for within the GEE analysis. There are several potential determinants which will be considered as covariates, such as age, parity, adherence, and SES. Relevant information will be collected at enrolment and throughout follow-up. Summary estimates (e.g., means, proportions, counts) will be reported with 95\% confidence intervals. Dichotomous outcomes will be compared using risk ratios with $95 \%$ confidence intervals, and the difference in continuous variables will be determined by comparing means. $P$ values less than 0.05 will be considered statistically significant. Statistical analyses will be conducted using Stata software. The plan for the analysis of secondary objectives and outcome measures will be presented elsewhere. 


\section{Discussion}

In this trial, we aim to assess the effectiveness of providing LSBE and MMN supplementation to non-pregnant adolescent and young women in rural Pakistan from preconception, and compare this intervention with the standard of care on anemia prevalence and other secondary health outcomes. The intervention will be implemented within the existing public health system, which is anticipated to lend to the interpretability of the trial findings and offer important insight around how to achieve coverage within the existing public health program context. This trial is distinctive in that it recruits both married and unmarried adolescent and young women, regardless of their intent to become pregnant, and collects measures on several domains of health and nutrition throughout participation.

The existing WHO guidelines on iron supplementation for non-pregnant adolescent and menstruating women can be confusing, particularly with respect to dosing frequency (weekly versus daily) and duration [27, 28]. At this time, we know of no guidelines that address preconception MMN supplementation. For adolescents, in particular, preconception care that includes $\mathrm{MMN}$ supplementation could be of benefit given that they are still developing and anemia can be caused by multiple different micronutrient deficiencies beyond iron [29, 30]. Including a culturally-tailored educational piece aimed at empowering the ability of participants to make informed decisions about their health, well-being, and nutrition will be key to sustained behavioural change throughout the life-course. Similarly, guidelines on pre-pregnancy nutritional counselling specific to adolescents are not known to exist, even though attending to adolescents' nutritional needs will be important in addressing intergenerational malnutrition, chronic disease, and poverty [31]. Appropriate preconception care could allow that adolescents enter reproductive adulthood with improved health and nutrition status prior to conception.

Overall, the MaPPS Trial is expected to offer insight into providing an intervention that includes both LSBE and MMN supplementation to adolescent and young women from preconception and for a prolonged duration of time. We anticipate that there would be future follow up of the adolescent and young women in this cohort, so as to understand their long-term reproductive and empowerment outcomes, as well as development and disease outcomes. We also intend to investigate the cost-effectiveness of the provision of the described intervention.

\section{Trial status}

As of May 2018, participants are still being enrolled to the trial.

\section{Additional file}

Additional file 1 Additional tables detailing secondary outcome measures and cut-points. The additional file includes 3 tables detailing secondary nutritional status, anthropometric, and empowerment outcome measures for participants. (DOCX $23 \mathrm{~kb}$ )

\begin{abstract}
Abbreviations
AKU: Aga Khan University; GEE: Generalized estimating equations; LHW: Lady health worker; MaPPS Trial: Matiari emPowerment and preconception supplementation trial; MMN: Multiple micronutrient; MUAC: Middle-upper arm circumference; SES: Socioeconomic status; UNIMMAP: UNICEF/ WHO/ UNU international multiple micronutrient preparation; WHO: World Health Organization
\end{abstract}

\section{Acknowledgements}

The authors wish to acknowledge the entire MaPPS Trial study team for their contributions to data collection, monitoring, logistics, and general study operation, particularly Qamaruddin Junejo (field supervisor). We would also acknowledge the collaboration of the following groups within District Matiari: the District of Health, Lady Health Worker Programme, and Education Department. We appreciate the ongoing assistance and support provided by Imran Ahmed, Arjumand Rizvi, Rasool Bux, and the members of the Data Management Unit at AKU; Didar Alam and members of the Nutrition Research Lab at AKU for ongoing laboratory coordination and logistics; and Mir Asghar Ali Khan and Ishrat Abbas for grant and budgetary assistance.

\section{Funding}

Financial support to conduct this trial was provided by the Bill \& Melinda Gates Foundation and the World Food Programme. Neither funding body had any role in the conduction of the study or the preparation of this manuscript.

\section{Authors' contributions}

ZAB conceived the trial and secured funding. JBB, ZAB, and SBS participated in the initial conceptualization, design, and writing of the study protocol. YW and ZS have contributed ongoing input around the design, study protocol development, and data collection methods. YW, SBS, and ZS have implemented the study in Matiari District. YW oversees field operations. JBB produced the first draft of this manuscript, with input from ZAB, YW, SBS, and ZS. All authors reviewed this manuscript and approved the final version. $Z A B$ is the guarantor.

\section{Ethics approval and consent to participate}

Ethics approval for this trial was obtained from the Aga Khan University Ethics Review Committee on August 16, 2016 (Number: 4324-Ped-ERC-16); and from the Research Ethics Board at the Hospital for Sick Children on November 17, 2016 (Number: 1000054682). All women enrolled in the trial are asked for their written consent to participate in the trial and are free to decline or stop their participation at any time without any consequences.

Competing interests

The authors declare that they have no competing interests.

\section{Publisher's Note}

Springer Nature remains neutral with regard to jurisdictional claims in published maps and institutional affiliations.

\section{Author details}

${ }^{1}$ Centre for Global Child Health, The Hospital for Sick Children, Toronto, ON M6S 1S6, Canada. '2Department of Nutritional Sciences, University of Toronto, Toronto, ON, Canada. ${ }^{3}$ Centre of Excellence in Women and Child Health, Aga Khan University, Stadium Road, Karachi 74800, Pakistan. 
Received: 14 May 2018 Accepted: 24 May 2018

Published online: 30 May 2018

\section{References}

1. Every Woman Every Child. Global Strategy for Women's, Children's and Adolescents Health 2016-2030. New York: Every Woman Every Child; 2015.

2. Sawyer SM, Afifi RA, Bearinger LH, Blakemore SJ, Dick B, Ezeh AC, Patton GC. Adolescence: a foundation for future health. Lancet. 2012;379(9826):1630-40.

3. World Health Organization. WHO guidelines on preventing early pregnancy and poor Reprod Health outcomes among adolescents in developing countries. Geneva: World Health Organization; 2011.

4. UNICEF. Adolescent Health. 2016. https://www.unicef.org/health/index_ 92029.html. Accessed 07 May 2018

5. Bhutta ZA, Lassi ZS, Bergeron G, Koletzko B, Salam R, Diaz A, McLean M, Black RE, De-Regil LM, Christian P, Prentice AM, Klein JD, Keenan W, Hanson M. Delivering an action agenda for nutrition interventions addressing adolescent girls and young women: priorities for implementation and research. Ann N Y Acad Sci. 2017;1393(1):61-71.

6. Black RE, Victora CG, Walker SP, Bhutta ZA, Christian P, de Onis M, Ezzati M, Grantham-McGregor S, Katz J, Martorell R, Uauy R. Maternal and child nutrition study group. Maternal and child undernutrition and overweight in low-income and middle-income countries. Lancet. 2013;382(9890):427-51.

7. Thompson B, Amoroso L. Combating Micronutrient Deficiencies: Foodbased approaches. Rome: Food and agricultural organization of the United Nations; 2010

8. Dean SV, Lassi ZS, Imam AM, Bhutta ZA. Preconception care: promoting reproductive planning. Reprod Health. 2014;11(Suppl 3):S2.

9. Dean SV, Lassi ZS, Imam AM, Bhutta ZA. Preconception care: nutritional risks and interventions. Reprod Health. 2014;11(Suppl 3):S3.

10. Chan AW, Tetzlaff JM, Gøtzsche PC, Altman DG, Mann H, Berlin JA, et al. SPIRIT 2013 explanation and elaboration: guidance for protocols of clinical trials. BMJ. 2013;346:e7586.

11. Baxter JB, Wasan Y, Soofi SB, Suhag Z, Bhutta ZA. Effect of life skills building education and micronutrient supplements provided from preconception versus the standard of care on low birth weight births among adolescent and young Pakistani women (15-24 years): a prospective, population-based cluster-randomized trial. Reprod Health. 2018.

12. Aga Khan University. National Nutrition Survey 2011. In: Government of Pakistan; 2013.

13. van Liere M, Bhutta ZA, Hartvig-Blomberg J, Mahmood S. Embodying the future: how to improve the nutritional status of adolescent girls in Pakistan? Global Alliance for Improved Nutrition; 2017.

14. Pakistan Strategy Support Program. Food security, consumption, poverty and nutrition in Pakistan: what do we know and what can we do about it Washington: International Food Policy Research Institute; 2014.

15. Hafeez A, Mohamud BK, Shiekh MR, Shah SA, Jooma R. Lady health workers programme in Pakistan: challenges, achievements and the way forward. J Pak Med Assoc. 2011;61:210-5.

16. World Health Organization. Global health workforce alliance. Pakistan's lady health worker Programme: Global Health workforce alliance, World Health Organization, case study. Geneva: World Health Organization; 2008.

17. Coates J, Swindale A, Bilinsky P. Household food insecurity access scale (HFIAS) for measurement of household food access: Indicator guide (v. 3). Washington: food and nutrition technical assistance project, academy for educational Development; 2007.

18. Currie C, Inchley J, Molcho M, Lenzi M, Veselska Z, Wild F. Health Behaviour in School-aged Children (HBSC) Study Protocol: Background, Methodology, and Mandatory items for the 2013/14 Survey. St. Andrews: CAHRU; 2014

19. Zimet GD, Dahlem NW, Zimet SG, Farley GK. The multidimensional scale of Percieved social support. J Pers Assess. 1988;52:30-41.

20. National Institute of Population Studies (NIPS) [Pakistan] and ICF International. Pakistan Demographic and Health Survey 2012-13. Islamabad and Calverton: NIPS and ICF International; 2013.

21. Schwarzer R, Jerusalem M. Generalized self-efficacy scale. In: Weinman J, Wright $\mathrm{S}$, Johnston $\mathrm{M}$, editors. Measures in health psychology: a user's portfolio. Causal and control beliefs. Windsor: NFER-NELSON; 1995. p. 35-7.

22. Cohen S, Williamson G. Perceived stress in a probability sample of the United States. In: Spacapan S, Oskamp S, editors. The social psychology of health: Claremont Symposium on applied Social Psychology. Newbury Park: Sage; 1988.
23. Lovibond SH, Lovibond PF. Manual for the Depression Anxiety Stress Scales. Sydney: Psychology Foundation; 1995

24. Straus MA. Measuring Intrafamily conflict and violence: the conflict tactics (CT) scales. J Marriage Fam. 1979:41:75-88

25. Gibson RS, Ferguson EL. An interactive 24-hour recall for assessing the adequacy of iron and zinc intakes in developing countries. Cali, Colombia: International Food Policy Research Institute (IFPRI) and International Center for Tropical Agriculture (CIAT); 2008.

26. Caulfield LE, Bose A, Chandyo RK, Nesamvuni C, de Moraes ML, Turab A, et al. Infant feeding practices, dietary adequacy, and micronutrient status measures in the MAL-ED study. Clin Infect Dis. 2014;59(Suppl 4):S248-54.

27. WHO. Guideline: Intermittent iron and folic acid supplementation in menstruating women. Geneva: World Health Organization; 2011.

28. WHO. Guideline: Daily Iron supplementation in adult women and adolescent girls. Geneva: World Health Organization; 2016.

29. Fishman SM, Christian P, West KP. The role of vitamins in the prevention and control of anaemia. Public Health Nutr. 2000;3(2):125-50.

30. Ramakrishnan U, Grant F, Goldenberg T, Zongrone A, Martorell R. Effect of women's nutrition before and during early pregnancy on maternal and infant outcomes: a systematic review. Paediatr Perinat Epidemiol. 2012; 26(Suppl 1):285-301.

31. Lassi ZS, Mansoor T, Salam RA, Bhutta SZ, Das JK, Bhutta ZA. Review of nutrition guidelines relevant for adolescents in low- and middle-income countries. Ann N Y Acad Sci. 2017;1393(1):51-60.

\section{Ready to submit your research? Choose BMC and benefit from:}

- fast, convenient online submission

- thorough peer review by experienced researchers in your field

- rapid publication on acceptance

- support for research data, including large and complex data types

- gold Open Access which fosters wider collaboration and increased citations

- maximum visibility for your research: over $100 \mathrm{M}$ website views per year

At BMC, research is always in progress.

Learn more biomedcentral.com/submissions 